OHSTPY-HEP-T-99-024

UMD-D-99-4

hep-th/9911243

\title{
The Mass Spectrum of $\mathcal{N}=1 \mathrm{SYM}_{2+1}$ at Strong Coupling
}

\author{
Paul Haney ${ }^{a}$, John R. Hiller ${ }^{b}$, Oleg Lunin ${ }^{a}$, \\ Stephen Pinsky ${ }^{a}, \mathrm{Uwe}$ Trittmann $^{a}$ \\ ${ }^{a}$ Department of Physics, \\ The Ohio State University, \\ Columbus, $\mathrm{OH}$ 43210, USA \\ ${ }^{b}$ Department of Physics, \\ University of Minnesota Duluth, \\ Duluth, MN 55812, USA
}

\begin{abstract}
We consider supersymmetric Yang-Mills theory on $\mathbf{R} \times S^{1} \times S^{1}$. In particular, we choose one of the compact directions to be light-like and another to be space-like. Since the SDLCQ regularization explicitly preserves supersymmetry, this theory is totally finite, and thus we can solve for bound state wave functions and masses numerically without renormalizing. We present the masses as functions of the longitudinal and transverse resolutions and show that the masses converge rapidly in both resolutions. We also study the behavior of the spectrum as a function of the coupling and find that at strong coupling there is a stable, well defined spectrum which we present. We also find several unphysical states that decouple at large transverse resolution. There are two sets of massless states; one set is massless only at zero coupling and the other is massless at all couplings. Together these sets of massless states are in one-to-one correspondence with the full spectrum of the dimensionally reduced theory.
\end{abstract}




\section{Introduction}

Recently, there has been considerable progress in understanding the properties of strongly coupled gauge theories with supersymmetry [1], 2, 3]. In particular, there are a number of supersymmetric gauge theories that are believed to be interconnected through a web of strong-weak coupling dualities. Although existing evidence for these dualities is encouraging, there is still an urgent need to address these issues at a more fundamental level. Ideally, we would like to solve for the bound states of these theories directly, and at any coupling.

Of course, solving a field theory from first principles is typically an intractable task. Nevertheless, it has been known for some time that $1+1$ dimensional field theories can be solved from first principles via a straightforward application of DLCQ (see [4] for a review). In more recent times, a large class of supersymmetric gauge theories in two dimensions was studied using a supersymmetric form of DLCQ (or 'SDLCQ'), which is known to preserve supersymmetry [7, 8, 9, 10, 11, 13, 14].

We have recently been able to extend the SDLCQ algorithms to solve higher-dimensional theories [5]. One important difference between two-dimensional and higher-dimensional theories is the phase diagram induced by variations in the gauge coupling. The spectrum of a $1+1$ dimensional gauge theory scales trivially with respect to the gauge coupling, while a theory in higher dimensions has the potential of exhibiting a complex phase structure, which may include a strong-weak coupling duality. Ref. [5] seemed to provide hints of the latter. It is therefore interesting to study the phase diagram of gauge theories in $D \geq 3$ dimensions.

Towards this end, we consider three dimensional $\mathrm{SU}\left(N_{c}\right) \mathcal{N}=1$ super-Yang-Mills compactified on the space-time $\mathbf{R} \times S^{1} \times S^{1}$. The calculations are all done in the large $N_{c}$ limit. In particular, we compactify the light-cone coordinate $x^{-}$on a light-like circle via DLCQ, and wrap the remaining transverse coordinate $x^{\perp}$ on a spatial circle. We are able to solve for bound state wave functions and masses numerically by diagonalizing the discretized light-cone supercharge. We have shown that the SDLCQ procedure extends naturally to $2+1$ dimensions, resulting in an exactly supersymmetric spectrum.

The contents of this paper are organized as follows. In Section 2, we formulate $\operatorname{SU}\left(N_{c}\right)$ $\mathcal{N}=1$ super-Yang-Mills defined on the compactified space-time $\mathbf{R} \times S^{1} \times S^{1}$. Explicit expressions are given for the light-cone supercharges, which are then discretized via the SDLCQ procedure. Quantization of the theory is carried out by imposing canonical 
(anti-)commutation relations for boson and fermion fields. We also discuss the two discrete symmetries of the theory in this section. In Section 3, we present our numerical results. We present plots of the spectrum as a function of the longitudinal and transverse resolution and show that it convergences very rapidly in both. We plot the spectrum also as a function of the coupling and find a very stable strong-coupling spectrum. We identify several states that appear to be unphysical and disappear at high transverse resolutions. Finally we discuss the infrared spectrum of the theory and present evidence that it is entirely determined by the dimensionally reduced theory. We conclude our analysis with a discussion of our results and a list of important related future projects in Section 4 .

\section{Light-Cone Quantization and SDLCQ}

Discrete light cone quantization has proven to be a very powerful method of studying the mass spectra of various theories [4]. It is well known that in $1+1$ dimension one can define a new version of DLCQ which preserves supersymmetry [7, 14]. In higher dimensions a supersymmetric prescription is also possible [5]. We begin by introducing light-cone coordinates $x^{ \pm}=\left(x^{0} \pm x^{1}\right) / \sqrt{2}$, and compactifying the $x^{-}$coordinate on a light-like circle. In this way, the conjugate light-cone momentum $k^{+}$is discretized. To discretize the remaining (transverse) momentum $k^{\perp}=k^{2}$, we may compactify $x^{\perp}=x^{2}$ on a spatial circle. Of course, there is a significant difference between the discretized light-cone momenta $k^{+}$and the discretized transverse momenta $k_{\perp}$; namely, the lightcone momentum $k^{+}$is always positive, while $k_{\perp}$ may take on positive or negative values. The positivity of $k^{+}$is a key property that is exploited in DLCQ calculations; for any given light-cone compactification, there are only a finite number of choices for $k^{+}-$ the total number depending on how finely we discretize the momenta. The 'resolution' of the discretization is usually characterized by a positive integer $K$, which is called the 'harmonic resolution' [15, 16]; for a given choice of $K$, the light-cone momenta $k^{+}$ are restricted to positive integer multiples of $P^{+} / K$, where $P^{+}$is the total light-cone momentum of a state. In the context of two-dimensional theories, this implies a finite number of Fock states [15].

In the case of interest here, we include an additional transverse dimension, and the number of Fock states is no longer finite, since there is an arbitrarily large number of transverse momentum modes defined on the transverse spatial circle. Thus, an additional truncation of the transverse momentum modes is required to render the total number 
of Fock states finite and the problem numerically tractable. This truncation procedure, which is characterized by a transverse resolution T, is analogous to the truncation of $k^{+}$ imposed by the 'harmonic resolution' $K$. Thus the transverse momentum $k_{\perp}$ range from zero to $\pm \frac{2 \pi T}{L}$, where $L$ is the size of the transverse circle.

Let us now review these ideas in the context of a specific super-Yang-Mills theory. We start with $(2+1)$-dimensional $\mathcal{N}=1$ super-Yang-Mills theory defined on a space-time with one transverse dimension compactified on a circle:

$$
S=\int d^{2} x \int_{0}^{L} d x_{\perp} \operatorname{tr}\left(-\frac{1}{4} F^{\mu \nu} F_{\mu \nu}+\mathrm{i} \bar{\Psi} \gamma^{\mu} D_{\mu} \Psi\right)
$$

After introducing the light-cone coordinates $x^{ \pm}=\frac{1}{\sqrt{2}}\left(x^{0} \pm x^{1}\right)$, decomposing the spinor $\Psi$ in terms of chiral projections

$$
\psi=\frac{1+\gamma^{5}}{2^{1 / 4}} \Psi, \quad \chi=\frac{1-\gamma^{5}}{2^{1 / 4}} \Psi
$$

and choosing the light-cone gauge $A^{+}=0$, we obtain

$$
\begin{aligned}
S=\int d x^{+} d x^{-} & \int_{0}^{L} d x_{\perp} \operatorname{tr}\left[\frac{1}{2}\left(\partial_{-} A^{-}\right)^{2}+\left(D_{+} \phi+\partial_{\perp} A^{-}\right) \partial_{-} \phi+\mathrm{i} \psi D_{+} \psi+\right. \\
& \left.+\mathrm{i} \chi \partial_{-} \chi+\frac{\mathrm{i}}{\sqrt{2}} \psi D_{\perp} \chi+\frac{\mathrm{i}}{\sqrt{2}} \chi D_{\perp} \psi\right]
\end{aligned}
$$

A simplification of the light-cone gauge is that the non-dynamical fields $A^{-}$and $\chi$ may be explicitly solved from their Euler-Lagrange equations of motion:

$$
A^{-}=\frac{1}{\partial_{-}^{2}} J=\frac{1}{\partial_{-}^{2}}\left(i g\left[\phi, \partial_{-} \phi\right]+2 g \psi \psi-\partial_{\perp} \partial_{-} \phi\right), \quad \chi=-\frac{1}{\sqrt{2} \partial_{-}} D_{\perp} \psi
$$

These expressions may be used to express any operator in terms of the physical degrees of freedom only. In particular, the light-cone energy, $P^{-}$, and momentum operators, $P^{+}, P^{\perp}$, corresponding to translation invariance in each of the coordinates $x^{ \pm}$and $x_{\perp}$ may be calculated explicitly:

$$
\begin{aligned}
P^{+} & =\int d x^{-} \int_{0}^{L} d x_{\perp} \operatorname{tr}\left[\left(\partial_{-} \phi\right)^{2}+\mathrm{i} \psi \partial_{-} \psi\right] \\
P^{-} & =\int d x^{-} \int_{0}^{L} d x_{\perp} \operatorname{tr}\left[-\frac{1}{2} J \frac{1}{\partial_{-}^{2}} J-\frac{\mathrm{i}}{2} D_{\perp} \psi \frac{1}{\partial_{-}} D_{\perp} \psi\right] \\
P_{\perp} & =\int d x^{-} \int_{0}^{L} d x_{\perp} \operatorname{tr}\left[\partial_{-} \phi \partial_{\perp} \phi+\mathrm{i} \psi \partial_{\perp} \psi\right] .
\end{aligned}
$$


The light-cone supercharge in this theory is a two component Majorana spinor, and may be conveniently decomposed in terms of its chiral projections:

$$
\begin{aligned}
Q^{+} & =2^{1 / 4} \int d x^{-} \int_{0}^{L} d x_{\perp} \operatorname{tr}\left[\phi \partial_{-} \psi-\psi \partial_{-} \phi\right] \\
Q^{-} & =2^{3 / 4} \int d x^{-} \int_{0}^{L} d x_{\perp} \operatorname{tr}\left[\partial_{\perp} \phi \psi+g\left(\mathrm{i}\left[\phi, \partial_{-} \phi\right]+2 \psi \psi\right) \frac{1}{\partial_{-}} \psi\right] .
\end{aligned}
$$

The action (3) gives the following canonical (anti-)commutation relations for propagating fields for large $N_{c}$ at equal $x^{+}$:

$$
\left[\phi_{i j}\left(x^{-}, x_{\perp}\right), \partial_{-} \phi_{k l}\left(y^{-}, y_{\perp}\right)\right]=\left\{\psi_{i j}\left(x^{-}, x_{\perp}\right), \psi_{k l}\left(y^{-}, y_{\perp}\right)\right\}=\frac{1}{2} \delta\left(x^{-}-y^{-}\right) \delta\left(x_{\perp}-y_{\perp}\right) \delta_{i l} \delta_{j k} .
$$

Using these relations one can check the supersymmetry algebra

$$
\left\{Q^{+}, Q^{+}\right\}=2 \sqrt{2} P^{+}, \quad\left\{Q^{-}, Q^{-}\right\}=2 \sqrt{2} P^{-}, \quad\left\{Q^{+}, Q^{-}\right\}=-4 P_{\perp} .
$$

We will consider only states which have vanishing transverse momentum, which is possible since the total transverse momentum operator is kinematical [. On such states, the light-cone supercharges $Q^{+}$and $Q^{-}$anticommute with each other, and the supersymmetry algebra is equivalent to the $\mathcal{N}=(1,1)$ supersymmetry of the dimensionally reduced (i.e. two-dimensional) theory [7]. Moreover, in the $P_{\perp}=0$ sector, the mass squared operator $M^{2}$ is given by $M^{2}=2 P^{+} P^{-}$.

As we mentioned earlier, in order to render the bound state equations numerically tractable, the transverse momentum of partons must be truncated. First, we introduce the Fourier expansion for the fields $\phi$ and $\psi$, where the transverse space-time coordinate $x^{\perp}$ is periodically identified:

$$
\begin{aligned}
& \phi_{i j}\left(0, x^{-}, x_{\perp}\right)= \\
& \frac{1}{\sqrt{2 \pi L}} \sum_{n^{\perp}=-\infty}^{\infty} \int_{0}^{\infty} \frac{d k^{+}}{\sqrt{2 k^{+}}}\left[a_{i j}\left(k^{+}, n^{\perp}\right) e^{-\mathrm{i} k^{+} x^{-}+\mathrm{i} \frac{2 \pi n^{\perp}}{L} x_{\perp}}+a_{j i}^{\dagger}\left(k^{+}, n^{\perp}\right) e^{\mathrm{i} k^{+} x^{-}-\mathrm{i} \frac{2 \pi n^{\perp}}{L} x_{\perp}}\right] \\
& \psi_{i j}\left(0, x^{-}, x_{\perp}\right)= \\
& \frac{1}{2 \sqrt{\pi L}} \sum_{n^{\perp}=-\infty}^{\infty} \int_{0}^{\infty} d k^{+}\left[b_{i j}\left(k^{+}, n^{\perp}\right) e^{-\mathrm{i} k^{+} x^{-}+\mathrm{i} \frac{2 \pi n^{\perp}}{L} x_{\perp}}+b_{j i}^{\dagger}\left(k^{+}, n^{\perp}\right) e^{\mathrm{i} k^{+} x^{-}-\mathrm{i} \frac{2 \pi n^{\perp}}{L} x_{\perp}}\right]
\end{aligned}
$$

Substituting these into the (anti-)commutators (10), one finds

$$
\left[a_{i j}\left(p^{+}, n_{\perp}\right), a_{l k}^{\dagger}\left(q^{+}, m_{\perp}\right)\right]=\left\{b_{i j}\left(p^{+}, n_{\perp}\right), b_{l k}^{\dagger}\left(q^{+}, m_{\perp}\right)\right\}=\delta\left(p^{+}-q^{+}\right) \delta_{n_{\perp}, m_{\perp}} \delta_{i l} \delta_{j k}
$$

\footnotetext{
${ }^{1}$ Strictly speaking, on a transverse cylinder, there are separate sectors with total transverse momenta $2 \pi n / L$; we consider only one of them, $n=0$.
} 
The supercharges now take the following forms:

$$
\begin{aligned}
& Q^{+}=\mathrm{i} 2^{1 / 4} \sum_{n^{\perp} \in \mathbf{Z}} \int_{0}^{\infty} d k \sqrt{k}\left[b_{i j}^{\dagger}\left(k, n^{\perp}\right) a_{i j}\left(k, n^{\perp}\right)-a_{i j}^{\dagger}\left(k, n^{\perp}\right) b_{i j}\left(k, n^{\perp}\right)\right], \\
& Q^{-}=\frac{2^{3 / 4} \pi \mathrm{i}}{L} \sum_{n^{\perp} \in \mathbf{Z}} \int_{0}^{\infty} d k \frac{n^{\perp}}{\sqrt{k}}\left[a_{i j}^{\dagger}\left(k, n^{\perp}\right) b_{i j}\left(k, n^{\perp}\right)-b_{i j}^{\dagger}\left(k, n^{\perp}\right) a_{i j}\left(k, n^{\perp}\right)\right]+ \\
& +\frac{\mathrm{i} 2^{-1 / 4} g}{\sqrt{L \pi}} \sum_{n_{i}^{\perp} \in \mathbf{Z}} \int_{0}^{\infty} d k_{1} d k_{2} d k_{3} \delta\left(k_{1}+k_{2}-k_{3}\right) \delta_{n_{1}^{\perp}+n_{2}^{\perp}, n_{3}^{\perp}} \\
& \times\left\{\frac{1}{2 \sqrt{k_{1} k_{2}}} \frac{k_{2}-k_{1}}{k_{3}}\left[a_{i k}^{\dagger}\left(k_{1}, n_{1}^{\perp}\right) a_{k j}^{\dagger}\left(k_{2}, n_{2}^{\perp}\right) b_{i j}\left(k_{3}, n_{3}^{\perp}\right)-b_{i j}^{\dagger}\left(k_{3}, n_{3}^{\perp}\right) a_{i k}\left(k_{1}, n_{1}^{\perp}\right) a_{k j}\left(k_{2}, n_{2}^{\perp}\right)\right]\right. \\
& +\frac{1}{2 \sqrt{k_{1} k_{3}}} \frac{k_{1}+k_{3}}{k_{2}}\left[a_{i k}^{\dagger}\left(k_{3}, n_{3}^{\perp}\right) a_{k j}\left(k_{1}, n_{1}^{\perp}\right) b_{i j}\left(k_{2}, n_{2}^{\perp}\right)-a_{i k}^{\dagger}\left(k_{1}, n_{1}^{\perp}\right) b_{k j}^{\dagger}\left(k_{2}, n_{2}^{\perp}\right) a_{i j}\left(k_{3}, n_{3}^{\perp}\right)\right] \\
& +\left(\frac{1}{k_{1}}+\frac{1}{k_{2}}-\frac{1}{k_{3}}\right)\left[b_{i k}^{\dagger}\left(k_{1}, n_{1}^{\perp}\right) a_{k j}^{\dagger}\left(k_{2}, n_{2}^{\perp}\right) a_{i j}\left(k_{3}, n_{3}^{\perp}\right)-a_{i j}^{\dagger}\left(k_{3}, n_{3}^{\perp}\right) b_{i k}\left(k_{1}\right) a_{k j}\left(k_{2}, n_{2}^{\perp}\right)\right]
\end{aligned}
$$

We now perform the truncation procedure; namely, in all sums over the transverse momenta $n_{i}^{\perp}$ appearing in the above expressions for the supercharges, we restrict summation to the following allowed momentum modes: $n_{i}^{\perp}=0, \pm 1 \ldots \pm T$. Note that this prescription is symmetric, in the sense that there are as many positive modes as there are negative ones. In this way we retain parity symmetry in the transverse direction.

How does such a truncation affect the supersymmetry properties of the theory? Note first that an operator relation $[A, B]=C$ in the full theory is not expected to hold in the truncated formulation. However, if $\mathrm{A}$ is quadratic in terms of fields (or in terms of creation and annihilation operators), one can show that the relation $[A, B]=C$ implies

$$
\left[A_{t r}, B_{t r}\right]=C_{t r}
$$

for the truncated operators $A_{t r}, B_{t r}$, and $C_{t r}$. In our case, $Q^{+}$is quadratic, and so the relations $\left\{Q_{t r}^{+}, Q_{t r}^{+}\right\}=2 \sqrt{2} P_{t r}^{+}$and $\left\{Q_{t r}^{+}, Q_{t r}^{-}\right\}=0$ are true in the $P_{\perp}=0$ sector of the truncated theory. The anticommutator $\left\{Q_{t r}^{-}, Q_{t r}^{-}\right\}$, however, is not equal to $2 \sqrt{2} P_{t r}^{-}$. So the diagonalization of $\left\{Q_{t r}^{-}, Q_{t r}^{-}\right\}$will yield a different bound-state spectrum than the one obtained after diagonalizing $2 \sqrt{2} P_{t r}^{-}$. Of course, the two spectra should agree in the limit $T \rightarrow \infty$. At any finite truncation, however, we have the liberty to diagonalize either of these operators. The choice of $\left\{Q_{t r}^{-}, Q_{t r}^{-}\right\}$specifies our regularization scheme. 
Choosing to diagonalize the light-cone supercharge $Q_{t r}^{-}$has an important advantage: the spectrum is exactly supersymmetric for any truncation. In contrast, the spectrum of the Hamiltonian $P_{t r}^{-}$becomes supersymmetric only in the infinite resolution limit.

Let us discuss the discrete symmetries of $Q^{-}$. There are three commuting $Z_{2}$ symmetries, one of them is the parity in the transverse direction:

$$
P: a_{i j}\left(k, n^{\perp}\right) \rightarrow-a_{i j}\left(k,-n^{\perp}\right), \quad b_{i j}\left(k, n^{\perp}\right) \rightarrow b_{i j}\left(k,-n^{\perp}\right) .
$$

Note that $P$ does not commute with $Q^{+}$or with $P_{\perp}$ :

$$
P Q^{+}=-Q^{+} P, \quad P P_{\perp}=-P_{\perp} P .
$$

The second symmetry is given by a generalization of the T-symmetry defined in [19] (we will call it $\mathrm{S}$ to avoid the confusion with transverse truncation parameter):

$$
S: a_{i j}\left(k, n^{\perp}\right) \rightarrow-a_{j i}\left(k, n^{\perp}\right), \quad b_{i j}\left(k, n^{\perp}\right) \rightarrow-b_{j i}\left(k,-n^{\perp}\right) .
$$

It commutes with all the Noether charges (5)-(9). Since $P$ and $S$ commute with each other one needs only one additional symmetry $R=P S$ to close the group.

Since $Q^{-}, P$ and $S$ commute with each other we can diagonalize them simultaneously. This allows us to diagonalization of the supercharge separately in the sectors with fixed $P$ and $S$ parities and thus will reduce the size of matrices. Doing this one finds that the roles of $P$ and $S$ are different. While all the eigenvalues are usually broken into nonoverlapping $S$-odd and $S$-even sectors [18], the $P$ symmetry leads to a double degeneracy of massive states (in addition to usual boson-fermion degeneracy due to supersymmetry). To demonstrate this, let us start from the massive bosonic state $|M+\rangle$ :

$$
\left(Q^{-}\right)^{2}|M+\rangle=M^{2}|M+\rangle, \quad P|M+\rangle=+|M+\rangle .
$$

If $M \neq 0$ then the bosonic state $Q^{+} Q^{-}|M+\rangle$ has nonzero norm and it is also an eigenstate of $\left(Q^{-}\right)^{2}$ with eigenvalue $M^{2}$. This state however has a negative parity:

$$
P Q^{+} Q^{-}|M+\rangle=-Q^{+} P Q^{-}|M+\rangle=-Q^{+} Q^{-} P|M+\rangle=-Q^{+} Q^{-}|M+\rangle .
$$

Thus we found one more nice feature of SDLCQ: in addition to preserving supersymmetry in the truncated theory it also preserves the degeneracy of the spectrum related to parity. We believe that this degeneracy will be lost in the usual DLCQ approximation but currently have no proof of that. 
To summarize, we have introduced a truncation procedure that facilitates a numerical study of the bound state problem, and preserves supersymmetry. The interesting property of the light-cone supercharge $Q^{-}$(14) is the presence of a gauge coupling constant as an independent variable, which does not appear in the study of two-dimensional theories. In the next section, we will study how variations in this coupling affects the bound states in the theory.

\section{$3 \quad$ Numerical Results}

In order to implement the DLCQ formulation of the bound-state problem - which is tantamount to imposing periodic boundary conditions $x^{-}=x^{-}+2 \pi R$ [16] - we simply restrict the light-cone momentum variables $k_{i}$ appearing in the expressions for $Q^{+}$and $Q^{-}$to the following discretized set of momenta: $\left\{\frac{1}{K} P^{+}, \frac{2}{K} P^{+}, \frac{3}{K} P^{+}, \ldots,\right\}$. Here, $P^{+}$ denotes the total light-cone momentum of a state, and may be thought of as a fixed constant, since it is easy to form a Fock basis that is already diagonal with respect to the operator $P^{+}$[15]. The reciprocal of the harmonic resolution $K$ measures the coarseness of our discretization. The continuum limit is then recovered by taking the limit $K \rightarrow \infty$. Physically, $1 / K$ represents the smallest positive unit of longitudinal momentum fraction allowed for each parton in a Fock state.

Of course, as soon as we implement the SDLCQ procedure, which is specified unambiguously by the harmonic resolution $K$, and cut off transverse momentum modes via the constraint $\left|n_{i}^{\perp}\right| \leq T$, the integrals appearing in the definitions for $Q^{+}$and $Q^{-}$are replaced by finite sums, and so the eigen-equation $2 P^{+} P^{-}|\Psi\rangle=M^{2}|\Psi\rangle$ is reduced to a finite matrix diagonalization problem. In this last step we use the fact that $P^{-}$is proportional to the square of the light-cone supercharge $Q^{-}$. Previously [5] we studied this theory with $K$ up to 5 , but with only one unit of transverse momentum corresponding to $T=1$. In Figure 11 we show the spectrum we obtained in that study as a function of a dimensionless coupling $g^{\prime}=g \sqrt{N L / 4 \pi^{3}}$. This figure shows several striking features that we want to analyze in more detail. First, we want to know whether the well defined strong-coupling spectrum, observed in Figure 1, persists at higher values of transverse resolution $T$ and study its convergence in $T$. The other striking feature of the $K=5$, $T=1$ spectrum is the state that is falling rapidly at large coupling. We will analyze the

\footnotetext{
${ }^{2}$ Strictly speaking, $P^{-}=\frac{1}{\sqrt{2}}\left(Q^{-}\right)^{2}$ is an identity in the continuum theory, and a definition in the compactified theory, corresponding to the SDLCQ prescription [7, 14].
} 


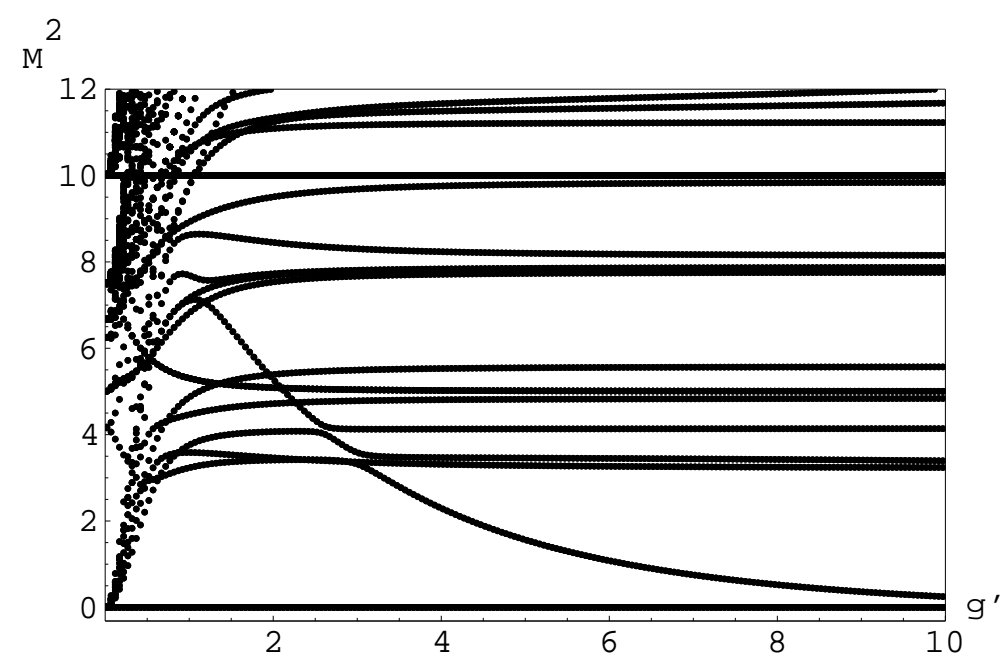

Figure 1: Plot of bound state mass squared $M^{2}$ in units of $2 \pi^{2} / L^{2}$ as a function of the dimensionless coupling $0 \leq g^{\prime} \leq 10$, defined by $\left(g^{\prime}\right)^{2}=g^{2} N L / 4 \pi^{3}$, at $N=1000$ and $K=5$. Note that there are massless states.

behavior of analogous states at different values of longitudinal and transverse resolutions and in particular we will be interested in the fate of the asymptotic (as $g \rightarrow \infty$ ) massless state in the continuum limit. In addition to this state there are many states that stay exactly massless at all values of coupling. As we have shown in [5], the number of such states does not depend on the value of the transverse resolution. The new numerical data support this result. The massive state at $g=0$ are just the discrete approximation the a continuum of free particles and is extactly calculable analytically.

Our previous SDLCQ calculations were done using a code written in Mathematica and performed on a PC. This code has now been rewritten in $\mathrm{C}++$ and some of the present work was done on supercomputers. We were able to perform numerical diagonalizations for $K=2$ through 7 and for values of $T$ up to $T=9$ at $K=4$ and $T=1$ at $K=7$.

\subsection{Strong Coupling: Massive Spectrum}

There are very few theories, other than in $1+1$ dimensions, where we have good information about the strong-coupling spectrum. In $1+1$ dimensions the concept of strong coupling has very little meaning since the coupling only comes in as an overall multiplicative constant in the Hamiltonian. There are a few lattice results but most of what 

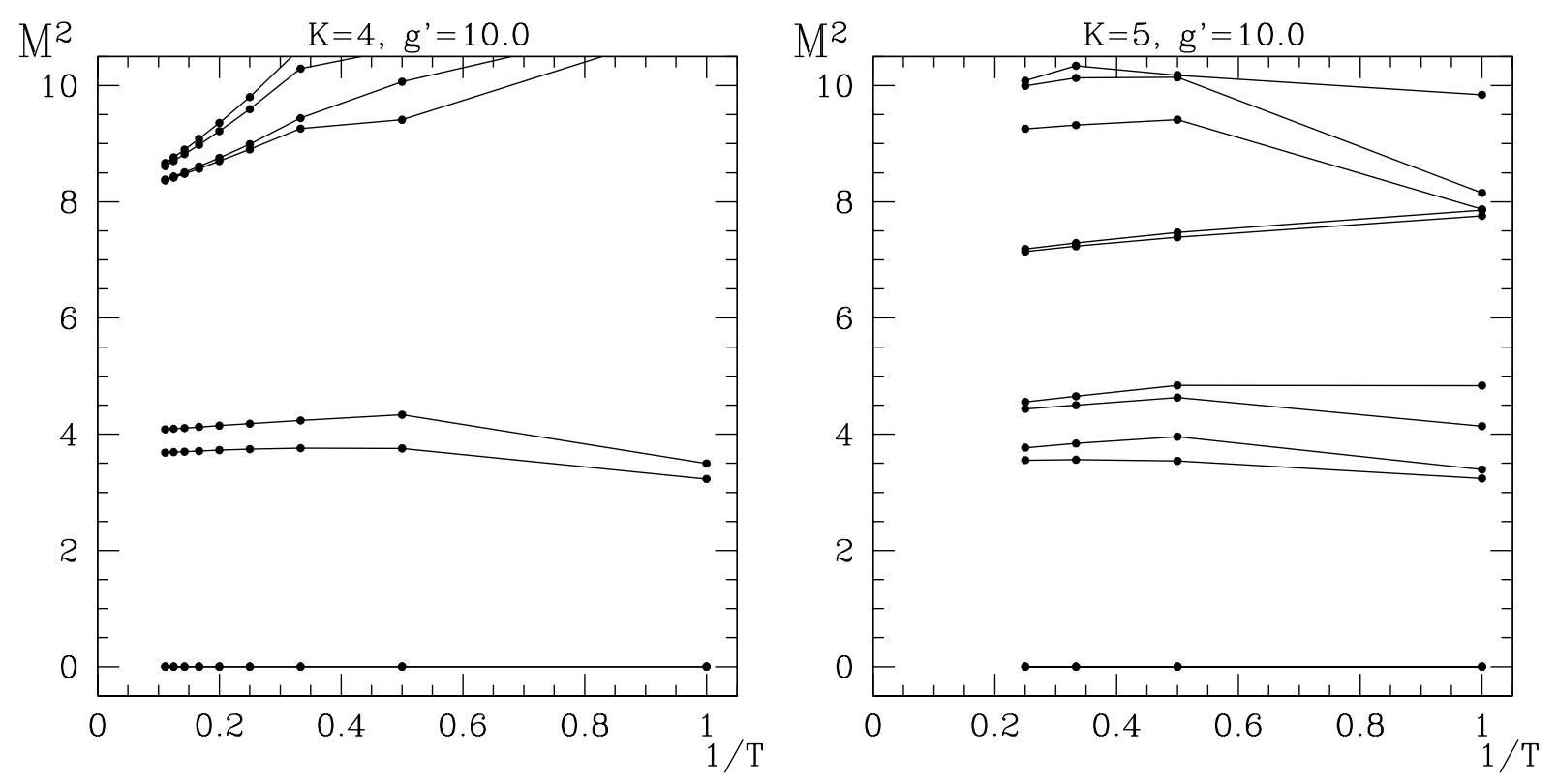

Figure 2: Plot of bound state mass squared $M^{2}$ in units of $2 \pi^{2} / L^{2}$ as a function of the transverse resolution $T$ for a coupling $g^{\prime}=10$ and for longitudinal resolutions $K=4$ (a) and $K=5$ (b). Boson and fermion masses are identical.

we know comes from theories that have dualities with weakly coupled theories. For the $(2+1)$-dimensional $\mathcal{N}=1$ SYM theory we are considering, there is no known duality between strong and weak coupling. Using SDLCQ, however, we can directly uncover the behavior of the theory at strong coupling.

In Figure 2, we plot the bound state mass squared $M^{2}$, in units of $4 \pi^{2} / L^{2}$, as a function of the transverse resolution $T$ for $K=4$ and $K=5$ in the strong-coupling regime. We see that these curves are amazingly flat, showing that this theory converges very rapidly with the transverse cutoff. We saw previously in $(1+1)$-dimensional models 9] that SDLCQ converges much faster than DLCQ, and this appears to persist for the transverse momentum in $2+1$ dimensions. From these figures it appears that we get sensible results by $T=2$ and good results already for $T=3$.

Given this rapid convergence, a sensible procedure is to remove the transverse cutoff by extrapolating the masses of the low lying bounds states to large transverse resolution at each value of the longitudinal resolution (thus constructing the SDLCQ spectrum of the complete three dimensional theory) and then extrapolating to large longitudinal resolution $\mathrm{K}$ for each of the states to find the spectrum as a function of the coupling. For $K=6$ and 7 we simply take the masses at the largest transverse momentum since there 

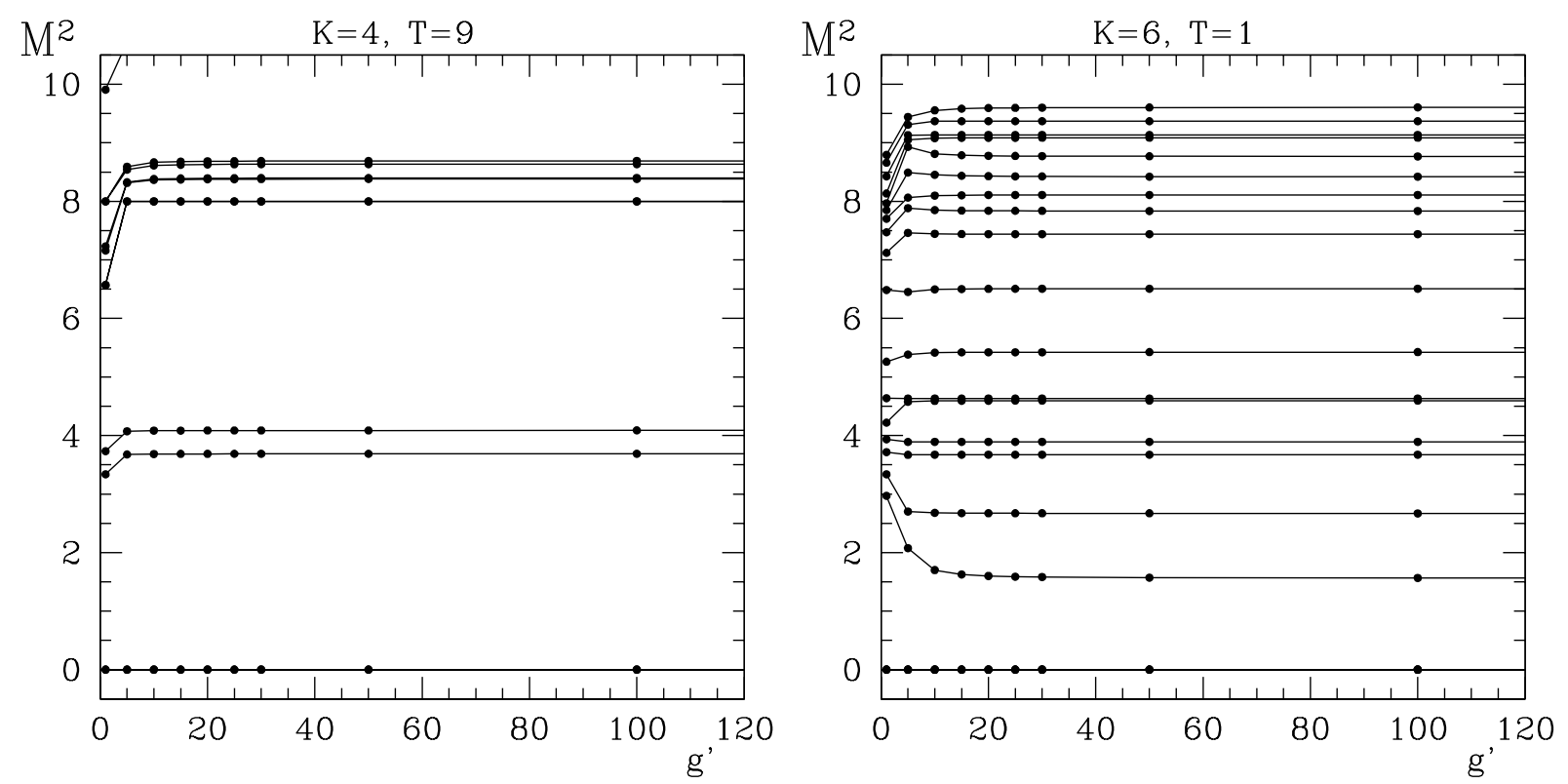

Figure 3: Plot of bound state mass squared $M^{2}$ in units of $4 \pi^{2} / L^{2}$ as a function of the coupling $g^{\prime}$. We show the plots for $K=4, T=9$ (a) and $K=6, T=1$ (b).

are not enough transverse moment points to make a meaningful extrapolation.

Let us look at the bound state mass as a function of the coupling. Fig. 3(a) shows an example of states completely settled down in transverse resolution, namely $K=4, T=9$. In contrast, Fig. 3(b) has only $T=1$ at $K=6$. Notice that nevertheless both plots show extremely stable states as a function of the coupling $g$, irrespective of their status of convergence in $T$. As we found in our preliminary study [5] of this theory, all the masses in the strong-coupling region are independent of the coupling. We see that by a coupling of $g^{\prime}=20$ a stable strong-coupling spectrum has appeared. We have looked as high as couplings of 1000 and we see this same behavior for all longitudinal and transverse resolutions.

In Figure 1 we plot the bound state mass as a function of $1 / K$. These results are the first calculation of the strong-coupling bound states of $\mathcal{N}=1 \mathrm{SYM}$ in $2+1$ dimensions. As we increase the resolution we are able to see states that have, as their primary component, more and more partons, and, as we have seen in other supersymmetric theories, many of these states appear at low energies. This accumulation of high-multiplicity low-mass states appears to be a unique property of SUSY theories. In non-SUSY theories the new states appear at increasing energies. In the dimensionally reduced version of this theory we saw that the accumulation point of these low-mass states appeared to be at zero mass 


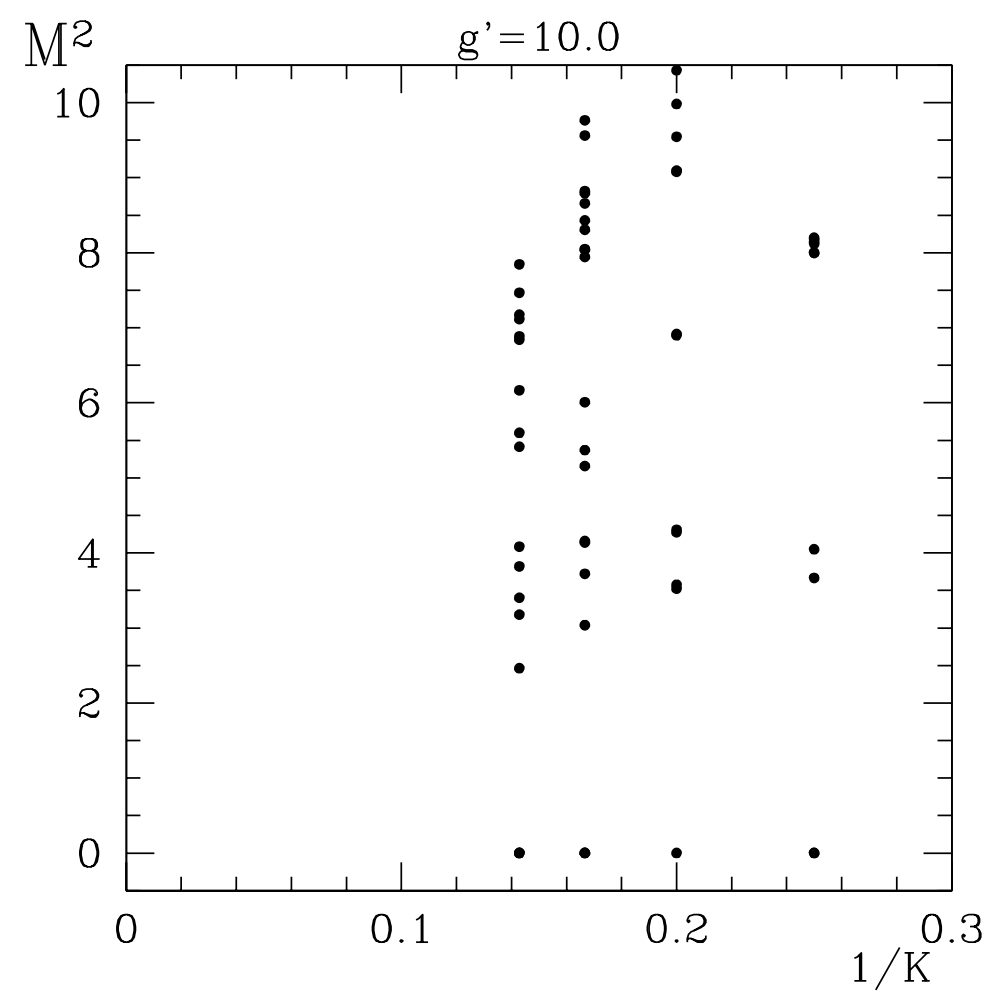

Figure 4: Plot of bound state mass squared $M^{2}$ in units of $2 \pi^{2} / L^{2}$ as a function of $1 / K$ for coupling $g^{\prime}=10$. For $K=3,4$ and 5 we plot the value of the mass obtained by extrapolating in the transverse resolution $T$. For $K=6$ and 7 we take the values at the highest resolution $T$.

[5, 6]. Here again we see clear evidence of an accumulation of low mass states, however we don't have sufficient information to say whether an accumulation point exists.

At this point we have not attempted to follow a specific bound state as a function of $K$. The appearance of new low mass as we increase $K$ makes this difficult, however a careful study of the wavefunction should make it possible. Also the accuracy of the extrapolation to infinite $K$ would be greatly impoved by addition of one or two additional transverse resolution at the largest values of $K$.

\subsection{Strong Coupling: Unphysical States}

In our preliminary study of this model we found one state that was particularly striking. It was very heavy at small coupling and approached zero mass at strong coupling, $c f$. Figure 11. We have now been able to look at this state at higher values of both transverse and longitudinal resolutions. We now believe that this state is most likely not a physical 


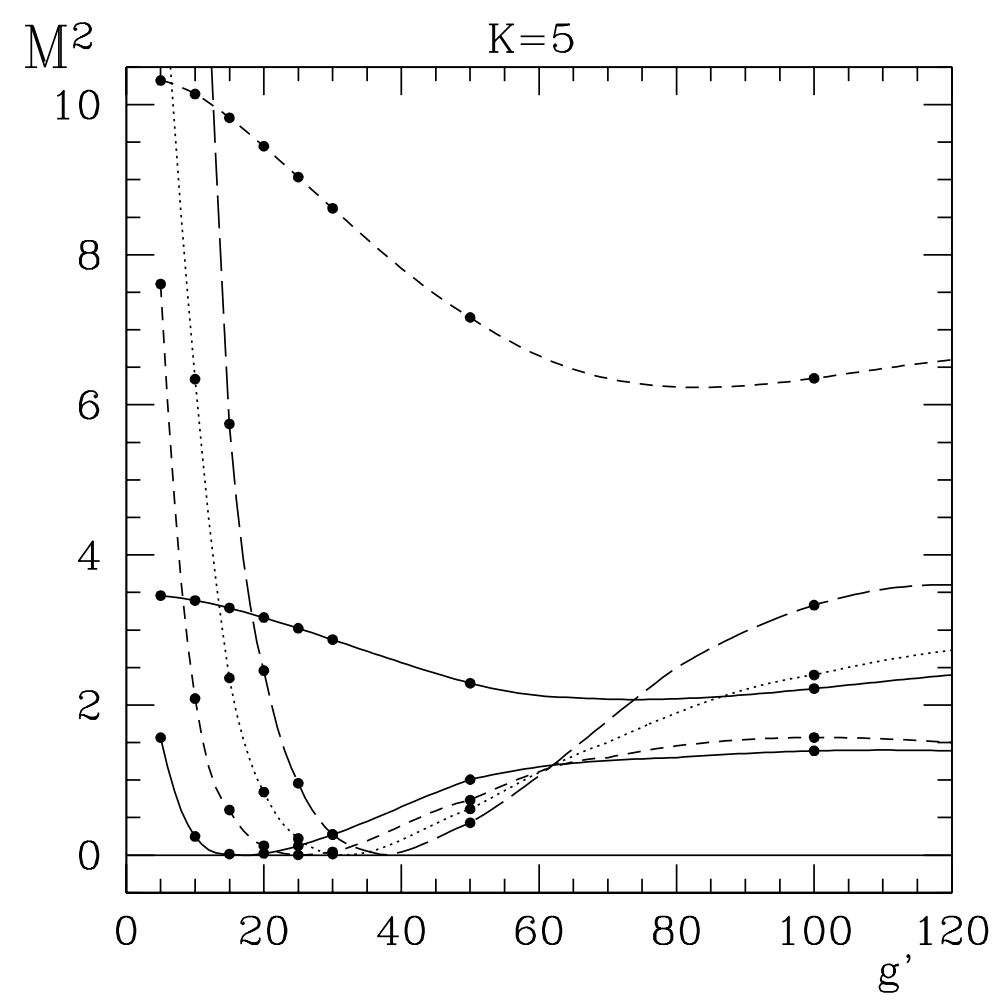

Figure 5: Plot of bound state masses falling rapidly with increasing coupling. The mass squared $M^{2}$ of these states in units of $4 \pi^{2} / L^{2}$ are plotted as a function of $g^{\prime}=g \sqrt{N L} / 4 \pi^{3 / 2}$ for $K=5$ at the transverse resolutions $T=1$ (solid lines), $T=2$ (dashed lines), $T=3$ (short dashed line) and $T=4$ (long dashed line).

one.

We are now able to identify two states with irregular coupling dependence which are shown in Figure 5 at various transverse resolution for $K=5$. We see that as we increase the transverse cutoff these states move up rapidly in mass, leading us to conclude that as $T \rightarrow \infty$, they decouple. One of these irregular states falls down to $M=0$ and then moves up, while another one has the same type of behavior, but with finite minimal mass. The fact that the first state touches zero is of some interest: a similar behavior was noted in a supersymmetric scalar matrix model in [8] and in that model the authors conjecture that this behavior might signal the existence of a critical coupling. Here the point where the mass goes to zero would appear to move to infinity as we remove the transverse cutoff, and it is not at all clear that those ideas carry over here.

At $K=6$ and $K=4$ we do not see these states, at least not at the same masses but at $K=7$ we again see such states. We believe that this is strong evidence that these 
are unphysical states. Clearly a real normalizable bound state must be visible at both even and odd resolutions, which is not the case here. Recently we have seen unphysical massless states of this type in a two dimensional theory with $(8,8)$ supersymmetry [6]. In that theory we had an independent theoretical evidence for them to be unphysical.

\subsection{Massless States}

In Figure 11 we see a number of states that become massless as $g^{\prime} \rightarrow 0$. We already explained this property in [5]. Namely at zero coupling only the first term survives in the supercharge (14) and then all the partons with $n_{\perp}=0$ (anti-)commute with $Q^{-}$. Thus any state constructed from such partons only becomes massless. The inverse statement is also true: at $g^{\prime}=0$ a massless state cannot contain any parton with $n_{\perp} \neq 0$. Thus the set of massless states at $g^{\prime}=0$ coincides with a Hilbert space of the theory dimensionally reduced to $1+1$. Moreover, the whole infrared spectrum of $\mathrm{SYM}_{2+1}$ at small coupling is governed by the dimensionally reduced theory (see [5] for details).

Previously [5, 10] we commented on the existence of exactly massless states and in [5] on the one-to-one correspondence between them and massless states of the $(1+1)$ dimensional model. Actually this fact provides an easy way to construct massless states for three-dimensional theories; the matrices to be diagonalized have a size much smaller than the ones used in the straightforward approach. The counting of massless states in three dimensions is also reduced to the analogous problem in $1+1$ dimensions. For finite $N_{c}$, even the $(1+1)$-dimensional case is not easily handled [10]; however, for large $N_{c}$ the multi-trace states decouple, and one needs to count only single-trace massless states. At resolution $K$ there are $2(K-1)$ of them. As a numerical check of the correspondence between massless sectors of $(2+1)$ and $(1+1)$-dimensional theories, we can count the massless states at different values of transverse resolution and as anticipated we found this number to be independent of $T$. These massless states are BPS states in the sense that they are destroyed by one super-charge, $Q^{-}$, and not the other, $Q^{+}$, and the BPS bound, which is zero here, is saturated.

In [5] it appeared that there were additional states that became massless as $g \rightarrow \infty$ but now we believe that these states are unphysical. Therefore the only massless states at nonzero coupling are the same $2(K-1)$ BPS states we saw in the dimensionally reduced model. 


\section{Discussion}

In this work, we considered the bound states of three dimensional $\mathrm{SU}(N) \mathcal{N}=1$ superYang-Mills defined on the compactified space-time $\mathbf{R} \times S^{1} \times S^{1}$. In particular, we compactified the light-cone coordinate $x^{-}$on a light-like circle via DLCQ, and wrapped the remaining transverse coordinate $x^{\perp}$ on a spatial circle. We showed explicitly that SDLCQ, employed in recent studies of $(1+1)$-dimensional supersymmetric gauge theories, extends naturally to $2+1$ dimensions. The supersymmetry becomes $\mathcal{N}=(1,1)$ because we can always chose $P_{\perp}$ to be zero in a light-cone quantized field theory, and SDLCQ provides a regularization scheme that preserves this supersymmetry. The supersymmetric theory considered here is finite and requires no renormalization.

By retaining a finite number of transverse and longitudinal modes, we were able to solve for bound-state wave functions and masses numerically by diagonalizing the discretized light-cone supercharge. The theory clearly has a stable spectrum at both small and large couplings. In Figure 2 we see that the theory converges very rapidly in the transverse resolution. We have seen in reference [10] that SDLCQ gives very smooth behavior in the longitudinal resolution. In Figure 5 we see that the states with irregular coupling dependence move off rapidly to high mass with increasing transverse resolution. They also do not seem to appear at resolutions 4 and 6 . We therefore conclude that they are not physical states. We see that there are no new massless states at strong coupling, and the complete massless sector of $\mathrm{SYM}_{2+1}$ is determined by the two-dimensional model. The number of exactly massless states at any coupling is $2(K-1)$, with no dependence on the transverse resolution. In addition, some states become massless as $g$ goes to zero, but their behavior is also described by the theory in $1+1$ dimensions. Consequently, we conclude that the entire massless spectrum of the $(2+1)$-dimensional model is determined by the dimensionally reduced model.

In previous work [5] we saw that the average number of particles in the massless states increases with $g$ and quickly becomes equal to the maximum number allowed by the resolution. We also see here that the number of low-mass states increases with resolution. Together this implies that at strong coupling the light states of this theory, and other SUSY theories, have a huge number of degrees of freedom. No doubt, it is this fact that allows for the possibility that these SUSY theories can contain all of the physics of dual theories in a different number of space-time dimensions. It would be interesting to relate this observation with the recent claim that strongly coupled super-Yang-Mills 
theory corresponds to string theory in an anti-de Sitter background [3]. Of course, the techniques we have employed here may be applied to any supersymmetric gauge theory defined on a suitably compactified space-time. This should facilitate a more general study of the strongly coupled dynamics of super-Yang-Mills theories, and in particular, allow us to scrutinize the string-like properties of Yang-Mills theories.

We have neglected the zero modes totally in this calculation. It remains an important problem to include these modes. We already know a great deal about zero modes [20] which are, after all, really only a few extra quantum mechanical degrees of freedom. It has recently been conjectured by Witten [22] that including these zero modes leads to spontaneous supersymmetry breaking in this theory.

Let us briefly comment on the $L$ dependence of the spectrum. For large $L$ the appropriate dimensionless $L$ independent mass scale is;

$$
\frac{M^{2}}{g^{4} N^{2}} \propto \frac{M^{2} L^{2} / 4 \pi^{2}}{\left(g^{\prime}\right)^{4}}
$$

At large $g^{\prime}$ the spectrum is constant in terms of $M^{2} L^{2} / 4 \pi^{2}$, therefore in terms of this dimensionless mass scale the entire spectrum would go to zero as $g^{\prime} \rightarrow \infty$. A possible interpretation of this result is that in the continuum limit this theory approaches a conformal field theory. There are however very massive states in our spectrum, that we did not study, that could remain massive in the contimuum limit.

The code that we are currently using is a newly written $\mathrm{C}++$ version of the Mathematica code that we used in much of our earlier work. Our large runs for matrix generation are currently performed at the Ohio Supercomputer Center. Matrix diagonalization is done using standard Lapack routines 21] on supercomputers at the Ohio Supercomputer Center and the Minnesota Supercomputing Institute. This is our first project with this new configuration, and we are currently working on several analytical and numerical improvements that we expect will allow us to increase by several factors of 10 the size of the problems we will be able to address in the future. Among these are $N=4 \mathrm{SYM}$ in $3+1$ and $N=1 \mathrm{SYM}$ in $2+1$ with a Chern-Simons term [22].

Acknowledgments This work is supported in part by the US Department of Energy. We would like to acknowledge the Ohio Supercomputer Center and the Minnesota Supercomputing Institute for grants of computing time. We also would like to acknowledge David G. Robertson of the Ohio Super Computing Center for his valued assistance and Aki Hashimoto for valuable conversations. 


\section{References}

[1] N. Seiberg, E. Witten, Nucl.Phys. B431 (1994) 484-550.

[2] N. Seiberg, Nucl.Phys. B435 (1995) 129-146.

[3] J.M. Maldacena, "The Large N Limit of Superconformal Field Theories and Supergravity," Adv.Theor.Math.Phys. 2 (1998) 231; hep-th/9711200.

[4] S.J. Brodsky, H.-C. Pauli, and S.S. Pinsky, Phys.Rept. 301 (1998) 299-486; hepph/9705477.

[5] F. Antonuccio, O. Lunin, S. Pinsky, Phys.Rev. D59 (1999) 085001; hepth/9811083

[6] F. Antonuccio, A. Hashimoto, O. Lunin, and S. Pinsky, JHEP 9907 (1999) 029; hep-th/9906087

[7] Y. Matsumura, N. Sakai, T. Sakai, Phys.Rev. D52 (1995) 2446.

[8] A. Hashimoto, I. R. Klebanov, Mod.Phys.Lett. A10 (1995) 2639.

[9] F. Antonuccio, O. Lunin, S. Pinsky, Phys.Lett. B429 (1998) 327-335; hepth/9803027

[10] F. Antonuccio, O. Lunin, S. Pinsky, Phys.Rev. D58 (1998) 085009; hepth/9803170

[11] F. Antonuccio, O. Lunin, H.-C. Pauli, S. Pinsky, and S. Tsujimaru, Phys.Rev. D58(1998) 105024; hep-th/9806133

[12] J.R. Hiller, O. Lunin, S. Pinsky and U. Trittmann, "The SDLCQ spectrum of Super Yang-Mills Theories in 1+1 Dimensions," in preparation.

[13] F. Antonuccio, H.-C. Pauli, S.Pinsky, and S. Tsujimaru, "DLCQ Bound States of $\mathcal{N}=(2,2)$ Super Yang-Mills at Finite and Large N," Phys.Rev. D58 (1998) 125006; hep-th/9808120

[14] F. Antonuccio, O. Lunin, and S. Pinsky, "On Exact Supersymmetry in DLCQ", Phys.Lett. B442 (1998) 173; hep-th/9809165 
[15] H.-C. Pauli and S.J. Brodsky, Phys.Rev. D32 (1985) 1993, 2001.

[16] T. Maskawa and K. Yamawaki, Prog.Theor.Phys. 56270 (1976).

[17] S. Dalley and I. Klebanov, Phys. Rev. D47 (1993) 2517-2527; hep-th/9209049.

[18] G. Bhanot, K. Demeterfi and I. Klebanov, Phys.Rev. D48 (1993) 4980-4990; hepth/9307111.

[19] D. Kutasov, Nucl. Phys. B414 (1994) 33.

[20] F. Antonuccio, O. Lunin, S. Pinsky, S. Tsujimaru, Phys.Rev. D60 (1999) 115006; hep-th/9811254

[21] E. Anderson et al., LAPACK Users' Guide, 3rd ed., (SIAM, Philadelphia, 1999).

[22] E. Witten, Supersymmetric Index of the Three-Dimensional Gauge Theory, IASSNS-HEP-99-20, hep-th/9903005 